\title{
14-3-3 Proteins regulate Akt Thr308 phosphorylation in intestinal epithelial cells
}

\author{
M Gómez-Suárez ${ }^{1,7}$, IZ Gutiérrez-Martínez ${ }^{1,7}$, JA Hernández-Trejo ${ }^{1,7}$, M Hernández-Ruiz², D Suárez-Pérez ${ }^{3,4}$, A Candelario ${ }^{1}$, \\ R Kamekura ${ }^{5}$, O Medina-Contreras ${ }^{6}$, M Schnoor ${ }^{2}$, V Ortiz-Navarrete ${ }^{2}$, N Villegas-Sepúlveda ${ }^{2}$, C Parkos ${ }^{5}$, A Nusrat ${ }^{5}$ and P Nava ${ }^{\star, 1}$
}

Akt activation has been associated with proliferation, differentiation, survival and death of epithelial cells. Phosphorylation of Thr308 of Akt by phosphoinositide-dependent kinase 1 (PDK1) is critical for optimal stimulation of its kinase activity. However, the mechanism(s) regulating this process remain elusive. Here, we report that 14-3-3 proteins control Akt Thr308 phosphorylation during intestinal inflammation. Mechanistically, we found that IFN $\gamma$ and TNF $\alpha$ treatment induce degradation of the PDK1 inhibitor, 14-3-3 $\eta$, in intestinal epithelial cells. This mechanism requires association of 14-3-3 $\zeta$ with raptor in a process that triggers autophagy and leads to 14-3-3 $\eta$ degradation. Notably, inhibition of 14-3-3 function by the chemical inhibitor BV02 induces uncontrolled Akt activation, nuclear Akt accumulation and ultimately intestinal epithelial cell death. Our results suggest that 14-3-3 proteins control Akt activation and regulate its biological functions, thereby providing a new mechanistic link between cell survival and apoptosis of intestinal epithelial cells during inflammation.

Cell Death and Differentiation (2016) 23, 1060-1072; doi:10.1038/cdd.2015.163; published online 5 February 2016

Mucosal cytokines alter epithelial homeostasis during inflammatory bowel diseases (IBD) by triggering several signaling pathways. Previously, we have reported that IFN $\gamma$ activates or inhibits epithelial cell proliferation by modulating $\beta$-catenin signaling pathway downstream of $\mathrm{Akt}^{1}$ in a mechanism that relies in the presence and phosphorylation of the scaffolding protein $14-3-3 \zeta^{2}$ The 14-3-3 family of adaptor proteins consists of seven members that associate with its client proteins mostly through phospho-serine/threonine motifs phosphorylated by serine/threonine kinases such as the AGC kinase Akt/PKB. ${ }^{3}$ Each 14-3-3 protein can interact with several targets and can also form homo or heterodimers with other family members in order to regulate biological processes, such as proliferation, differentiation and apoptosis. ${ }^{3,4}$ Therefore, 14-3-3 proteins are likely an important part of the machinery that regulates intestinal epithelial cell (IEC) homeostasis during physiological and pathological conditions.

Akt proteins (also known as PKB) are serine-threonine kinases that regulate cell survival, proliferation, metabolism and migration. ${ }^{5}$ Akt family encompasses three highly conserved proteins: Akt1, Akt2 and Akt3 that are the primary downstream mediators of $\mathrm{PIBK}^{6}$ All three Akt isoforms possess a pleckstrin homology $(\mathrm{PH})$ domain at the $\mathrm{N}$-terminal region, followed by a central kinase domain and a C-terminal regulatory domain. ${ }^{6}$ Full Akt activation requires direct interaction of its $\mathrm{PH}$ domain with Ptdlns $(3,4,5) \mathrm{P} 3$ that is mainly enriched at microdomains of the plasma membrane. ${ }^{6}$ At the plasma membrane, Akt is phosphorylated in two key residues, Threonine (Thr)308 (activation loop) and Serine (Ser)473 (C-terminal extension). Phosphorylation of Akt at Thr308 is mediated by phosphoinositide-dependent kinase 1 (PDK1), a serine/threonine kinase that phosphorylates several AGC kinase family members, including p70S6 kinase, p90S6 kinase and Akt. $^{7}$ PDK1 activation requires auto-phosphorylation of the residue Ser241 and is regulated by its association with several proteins including: $\mathrm{HSP}{ }^{8},{ }^{8} \mathrm{RSK}^{9}$ and $14-3-3$ family members. ${ }^{10}$ By contrast, the phosphorylation of Akt at Ser473 is mediated mainly by mTORC $2,{ }^{11}$ but can also be achieved by other kinases, including integrin-linked kinase (ILK) ${ }^{12}$ protein kinase C (PKC $\left.\varepsilon\right)^{13}$ and by auto-phosphorylation. ${ }^{14}$ Phosphorylation of Thr308 has been proposed to be a critical regulatory event for Akt activation. ${ }^{15}$ Once activated, Akt phosphorylates numerous substrates throughout the cell to regulate multiple cellular events and processes such as proliferation, differentiation and cell survival.

Deregulation of Akt kinases is frequently observed in human diseases such as cancer and diabetes; ${ }^{6}$ and our previous results have shown that Akt1 affects cell proliferation and

\footnotetext{
${ }^{1}$ Departamento de Fisiología, Biofísica y Neurociencias del CINVESTAV, IPN. Av. IPN 2508, Col. San Pedro Zacatenco, México, DF CP07360, México; ${ }^{2}$ Departamento de Biomedicina Molecular del CINVESTAV, IPN. Av. IPN 2508, Col. San Pedro Zacatenco, México, DF CP07360, México; ${ }^{3}$ Departamento de Morfología, Escuela Nacional de Ciencias Biológicas, IPN. Plan de Ayala s/n, Col. Santo Tomás, México, DF, México; ${ }^{4}$ PROINMED, FES Iztacala, UNAM, Avenida de los Barrios \#1, Col. Los Reyes Iztacala, Tlalnepantla, Estado de México, México; ${ }^{5}$ Department of Pathology, University of Michigan, Ann Arbor, 48109 Ml, USA and ${ }^{6}$ Laboratorio de Inmunología y Proteómica, Hospital Infantil de México 'Federico Gómez', México, DF 06720, México

${ }^{*}$ Corresponding author: P Nava, Departamento de Fisiología, Biofísica y Neurociencias del CINVESTAV, IPN, Av. IPN 2508, Col. San Pedro Zacatenco, México, DF CP07360, México. Tel: +52 5557473800; Fax: +404 727 3321; E-mail: pnava@fisio.cinvestav.mx

${ }^{7}$ These authors contributed equally to this work.

GS-M, GM-IZ, HT-JA, HR-M, C-A, K-R, MC-O and N-P performed experimental procedures, data interpretation and prepared images. SP-D and N-P performed immunofluorescences. GM-IZ, HT-JA, HR-M and N-P performed animal procedures. S-M, VS-N, ON-V, P-C, N-A and N-P supervised research and project planning, data interpretation and preparation of the manuscript.

Abbreviations: GSK3, glycogen synthase kinase 3; Lef, lymphoid enhancer factor; NLS, nuclear localization signal; Tcf, T-cell factor; IEC, intestinal epithelial cell; IFN interferon gamma; PDK1, phosphoinositide-dependent kinase 1; TNF $\alpha$, tumor necrosis factor alpha; PH, Plekstrin homology; CQ, chloroquine

Received 10.6.15; revised 14.11.15; accepted 24.11.15; Edited by RA Knight; published online 05.2.16
} 
survival in IECs during inflammation. ${ }^{1,2}$ But, while numerous studies have been focused in analyzing the mechanisms by which Akt kinases regulate cell homeostasis, ${ }^{1,2,5,16,17}$ the machinery controlling Akt activation during inflammatory disorders remains incompletely understood.

Here, we report that during inflammation 14-3-3 proteins control Akt1 activation in IECs by inhibiting its phosphorylation at Thr308. Our results demonstrate that cytokine exposure induces degradation of the PDK1 inhibitor, 14-3-3n. This resulted in PDK1 activation and facilitates phosphorylation of Akt Thr308 by PDK1. Interestingly, our findings also suggested that degradation of 14-3-3n was partially mediated by an autophagic process triggered by the association of the monomeric form of $14-3-3 \zeta$ (p14-3-3ZS58) with raptor. Inhibition of 14-3-3 function during colitis by the chemical inhibitor BV02 enhanced Akt activation, the accumulation of active Akt in the nucleus and ultimately augmented IEC death. Our findings describe an unexpected role for 14-3-3 proteins in controlling Akt phosphorylation. This mechanism could be important for fine-tuning Akt function during inflammation and maybe in the future exploited to identify new therapies aimed to control inflammatory diseases.

\section{Results}

Akt signaling pathway is activated in IECs of colitic mice. Inhibition of IEC proliferation by Akt contributes to the mucosal damage observed during inflammation. ${ }^{1}$ The mechanisms controlling Akt activity during this process remain unknown. We therefore analyzed Akt signaling pathway in the mucosa of C57BL/6J mice treated with dextran sulfate sodium (DSS), a well-known colitis model of epithelial injury. ${ }^{18}$ As reported previously by $u s,{ }^{19}$ a gradual decrease in cell proliferation, increased disease activity index (DAI) (Supplementary Figure 1A) and colon length shortening (Supplementary Figure 1B) were observed in DSS-treated mice. Moreover, Akt activation augments as revealed by the presence of the active form of Akt (phospho-Akt Thr308; pAkt308) (Figure 1a). ${ }^{1}$ Increased Akt activation was accompanied by phosphorylation of its downstream targets $\beta$-catenin ser552 ( $p \beta$-cat552) ${ }^{20}$ and GSK3 $\beta$ Ser9 (pGSK3 $\left.\beta\right)^{21}$ (Figure 1a). Augmented Akt1 levels were also detected in the mucosa of inflamed mice, but no changes in total $\beta$-catenin and GSK3 $\beta$ were observed (Figure 1a). Furthermore, pAkt308, p $\beta$-cat552 and pGSK3 $\beta$ presence was detected mainly in epithelial cells along the whole crypt axis in the mucosa of colitic mice (Figure 1b, white arrows). Akt full activation has been linked to its phosphorylation in several residues: the Serine473 (pAkt473), the Threonine308 (pAkt308), ${ }^{11}$ the Threonine450 (pAktT450) ${ }^{22}$ and the Serine 246 (pAktS246). ${ }^{23}$ Therefore, we analyzed the phosphorylation of Akt in those residues in samples of mice that were exposed to DSS for 1-4 days. pAkt308 levels increased with the DSS treatment, but its levels oscillated during the course of the experiment (Figures 1c and d). In contrast, DSS treatment augmented pAkt473 in a more constant level (Figures 1c and d). panAkt protein levels showed small variations (Figure 1c). Next, we analyzed the status of pAkt308 and pAkt473 in cell lysates of SW480 cells that were treated with IFN $\gamma$ for 1-12 h. As shown in Figure 1e, phosphorylation of Akt at 473 and 308 was detected as early as $1 \mathrm{~h}$ and remained elevated until $12 \mathrm{~h}$ after cytokine exposure. However, similar to our in vivo observations, pAkt473 and pAkt308 displayed different expression patterns (Figure 1f). panAkt protein levels remained unchanged (Figures 1c and e).

Next, pAkt450 and pAkt246 levels were analyzed in the colonic cell lysates obtained from the mice treated with DSS for 1-4 days. As shown in Figure 2a, pAkt450 and pAkt246 displayed a random expression pattern in our in vivo model. Meanwhile, pAkt450 increased abruptly 1 day post treatment and decreased slowly over time, pAkt246 protein levels decreased since day 1 (Figure 2a). To corroborate these findings, we next analyzed the presence of pAkt450 and pAkt246 in cell lysates of SW480 cells that were exposed to IFNy for 1-24 h. As shown in Figure 2b, pAkt450 levels marginally increased over time in the cells exposed to cytokines; meanwhile, pAkt246 presence was reduced. Taken together, these results suggested that during inflammation the phosphorylation of Akt at Ser473 and Thr308, but not at pAkt450 and pAkt246, was responsible for the activation of Akt in IECs during inflammation.

14-3-3n Regulates Akt full activation in IECs during colitis development. Akt phosphorylation at Ser473 has been extensively studied as a correlate for Akt activity; ${ }^{11-13,24,25}$ however, the mechanisms controlling the phosphorylation of Thr308 are rarely assessed. Thus, the machinery involved in the phosphorylation of Akt at the residue Thr308 during colitis was investigated. Akt Thr308 phosphorylation is mediated by PDK1. ${ }^{7}$ Pdk1 auto-phosphorylation at Ser241 (pPDK1) is critical for its activity; and therefore, PDK1 is considered to be a 'constitutively active' kinase. ${ }^{26}$ Thus, we investigated the status of pPDK1 in mice treated with DSS. As shown in Figure $3 a$, augmented pPDK1 levels were detected in the mucosa of colitic mice, but no changes in PDK1 levels were noticed. PDK1 auto-phosphorylation/activation is inhibited by its association with 14-3-3 proteins. ${ }^{10}$ Therefore, we analyzed the effect of overexpressing several 14-3-3 family members on its activation. As shown in Figure $3 b, 14-3-3 \eta$ but not 14-3-3 $\theta, \beta, \varepsilon$ or $\zeta$ inhibited PDK1 activation in SW480 cells as revealed by the presence of pPDK1. The inhibitory effect of $14-3-3 \eta$ on PDK1 is well characterized; ${ }^{10}$ therefore, we analyzed the presence of $14-3-3 \eta$ in the mucosa of colitic mice. As shown in Figure 3c, low levels of 14-3-3n and slight upregulation of $14-3-3 \theta$ were detected in mucosal cell lysates of colitic mice as compared with control mice. No changes in protein levels for $14-3-3 \beta, 14-3-3 \varepsilon$ and $14-3-3 \zeta$ were seen (Figure $3 c$ ). Densitometric analysis confirmed that 14-3-3n was diminished and pPDK1 increased during colitis (Figure $3 d$ ). No changes in $14-3-3 \zeta$ protein levels were perceived (Figure 3d). Moreover, immunofluorescence analysis revealed that in control animals $14-3-3 \eta$ and pPDK1 were present along the whole crypt-surface axis, but meanwhile $14-3-3 \eta$ was enriched at the crypt base, pPDK1 was mainly expressed at the upper third of the colonic crypts (Figure 3e). However, in the mucosa of colitic mice 14-3-3n was decreased and pPDK1 augmented (Figure 3e). Taken 
a

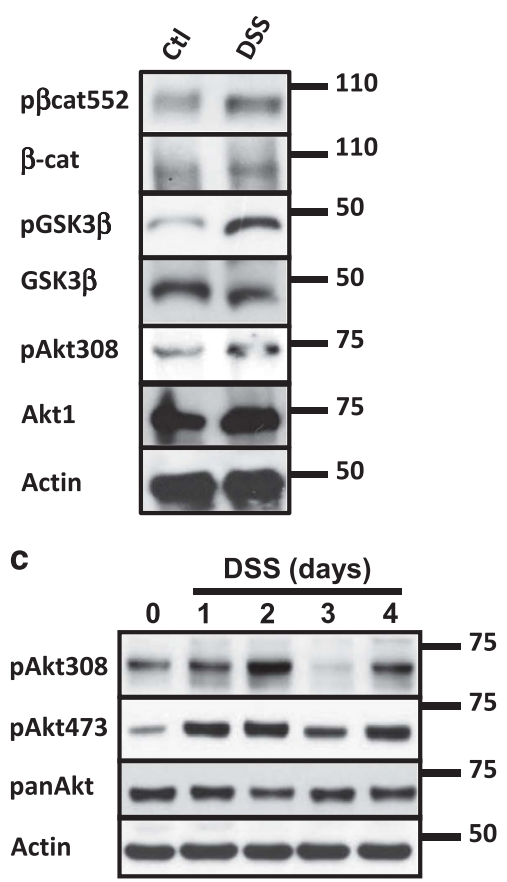

e

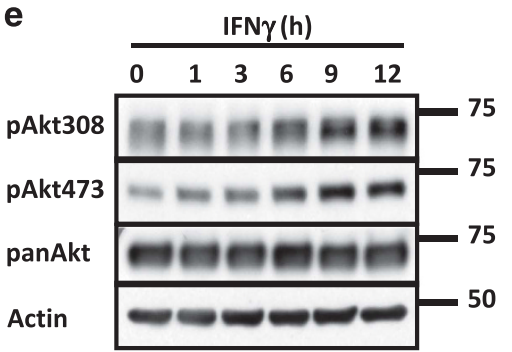

b
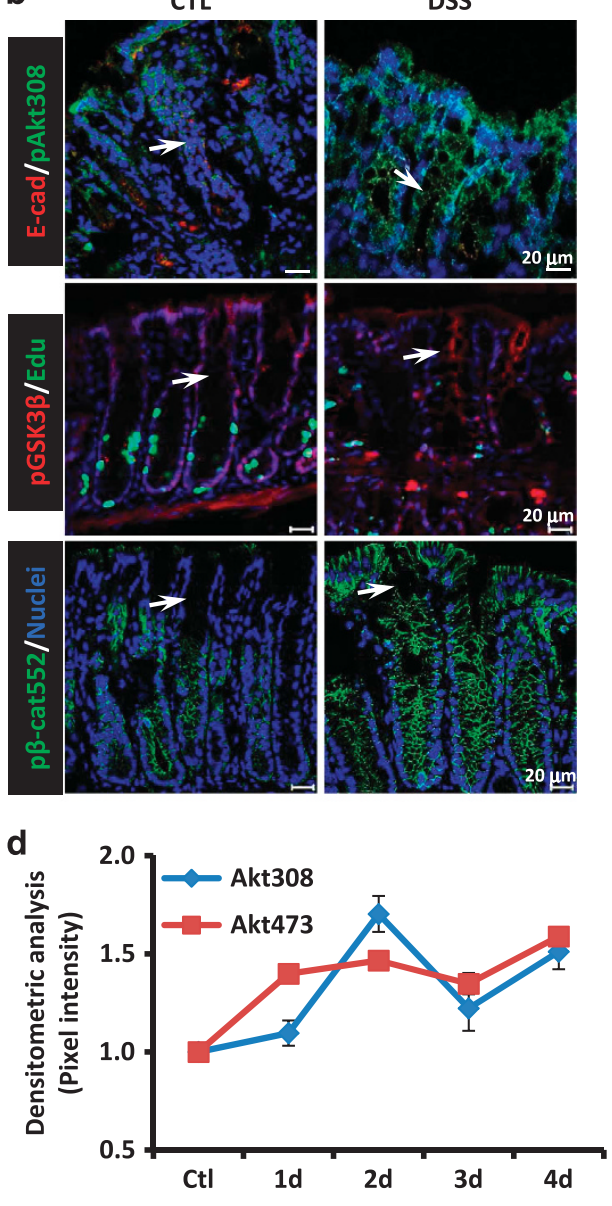

f

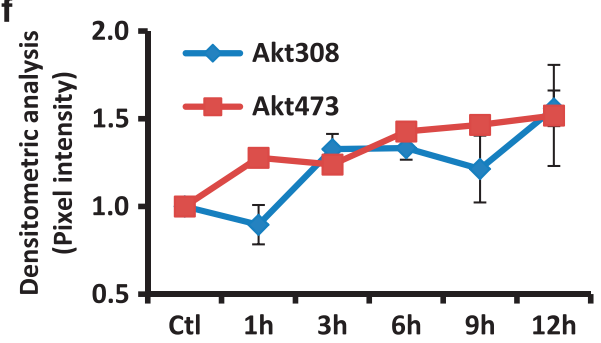

Figure 1 Akt signaling pathway is activated in IECs by proinflammatory cytokines. (a) pGSK3 $\beta$, GSK3 $\beta, p \beta$-cat552, $\beta$-catenin, pAkt473, pAkt308 and Akt1 were analyzed by western blotting in mucosal cell lysates of C57BL/6J mice. C57BL/6J received drinking water or drinking water with DSS (3\%) for 4 days. Actin was used as a control. (b) Distribution of E-cadh (red), pAkt308 (Green), pGSK3 $\beta$ (red), EdU (green) and p $\beta$-cat552 (green) was examined by immunofluorescence in the mucosa of control and DSStreated animals. C57BL/6J received drinking water or drinking water with DSS (3\%) for 4 days. White arrow marks cell borders of IECs. Nuclei are blue. Bar $=20 \mu \mathrm{m}$. (c) Phosphorylation of Akt at Serine 473 and Threonine 308 and panAkt levels were analyzed in the mucosa of mice treated with DSS. C57BL/6J received drinking water or drinking water with DSS (3\%) for 1-4 days. Actin was used as a loading control. (d) Densitometric analysis of western blots obtained for pAkt at S473 and pAkt T308 was performed and graphs were shown $(n=4)$. (e) Phosphorylation of Akt at 473 and 308 and panAkt protein levels were investigated in SW480 cells treated with IFN (100 U/ml) for 1-12 h. Actin was used as a loading control. (f) Densitometric analysis of the western blots obtained for pAkt473 and pAkt308 in SW480 cells treated with IFN $\gamma$ (100 U/ml) is shown in the graph $(n=3)$

together, these results suggested that $14-3-3 \eta$ could negatively regulate PDK1 activity during inflammation.

p14-3-3 $\zeta 58$ induces $14-3-3 \eta$ degradation and regulates Akt activation. Most cellular proteins in eukaryotic cells are degraded via two major pathways: the proteasomal and the lysosomal system. Although the first one degrades the majority of proteins, the second one is responsible for degradation of aggregated proteins and cellular organelles. ${ }^{27}$ Therefore, we investigated whether 14-3-3n degradation after cytokine treatment was dependent on any of those mechanisms. As shown in Figure 4a, 14-3-3n levels diminished in cells treated with cytokines, but inhibition of lysosomal (Chloroquine; CQ) or proteasomal (MG132) degradation systems partially rescued the reduction in 14-3-3n induced by the cytokines. In contrast, calpain inhibition by ALLN did 
not affect $14-3-3 \eta$ cytokine-mediated degradation (Figure 4a). These results suggested that $14-3-3 \eta$ undergoes lysosomal and proteasomal degradation in IECs treated with proinflammatory cytokines.

Proinflammatory cytokines promote autophagy-mediated clearance of unnecessary proteins through lysosomal degradation. $^{28,29}$ This led us to speculate that autophagymediated degradation of $14-3-3 \eta$ was occurring during a
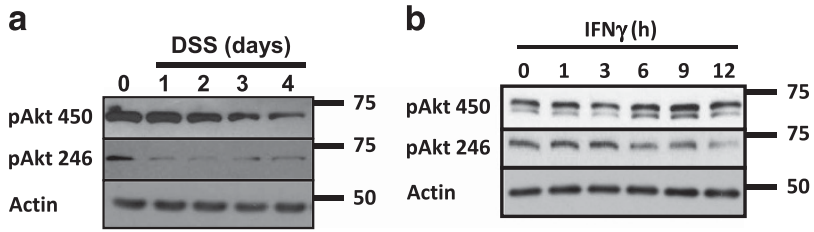

Figure 2 The phosphorylation of Akt Thr450 and Akt Ser246 is affected by inflammatory stimulus. (a) The effects of DSS-induced inflammation on pAktThr450 and pAktSer246 were analyzed in mucosal samples of C57BL/6J mice by western blotting. C57BL/6J mice were treated with DSS (3\%) for 1-4 days. Actin was used as a control $(n=7)$. (b) The presence of pAktThr450 and pAktSer246 was analyzed by western blotting in cell lysates of SW480 cells treated with IFN $\gamma(100 \mathrm{U} / \mathrm{ml})$ for $1-12 \mathrm{~h}$. Actin was used as a control $(n=3)$ inflammation. We investigated the presence of autophagy in the mucosa of colitic mice by analyzing the conversion of the autophagosomal marker LC3-I to LC3-II ${ }^{31,32}$ and the presence of ATG5, a protein that is essential for autophagosome formation. ${ }^{30}$ As shown in Figure $4 \mathrm{~b}$, the amount of LC3-II and ATG5 augmented in mucosal samples obtained from DSS-treated mice. Moreover, immunofluorescence assays revealed that LC3-II and the autophagosomal marker p62 $2^{31}$ were enriched in vesicles present in IECs (white arrow) and in cells located at the lamina propria (red star; control mice). The number of both cell types was increased in the mucosa of colitic mice (Figures 4c and d). Next, we evaluated autophagy in SW480 cells that were exposed to IFN $\gamma /$ TNF $a$ for $1-24 \mathrm{~h}$ by investigating LC3 I-II conversion. As shown in Supplementary Figure 2A (upper panel), LC3-II levels augmented in cells treated with cytokines. Moreover, autophagolysosomes detection with acridine orange confirmed that cytokines treatment triggered autophagy in IECs (Supplementary Figure 2A; lower panel). Next, 14-3-3 $\eta$ and PDK1 activation was analyzed in the mucosa of colitic mice treated with vehicle alone or treated with $C Q$. As shown in Figure 4e, 14-3-3n was reduced in the mucosa of DSS-treated mice; and its reduction was accompanied by PDK1 activation and $C Q$ treatment prevented this a

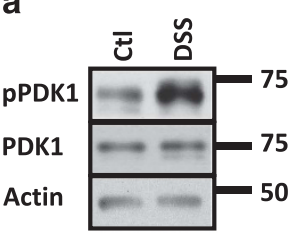

b

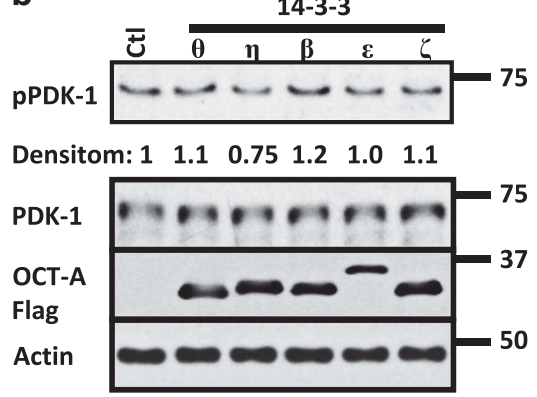

C

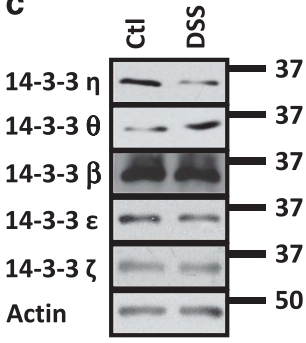

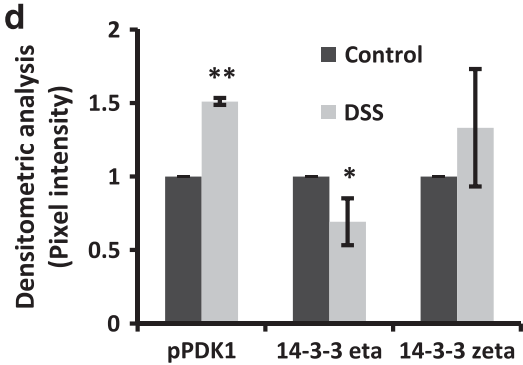

e CtI
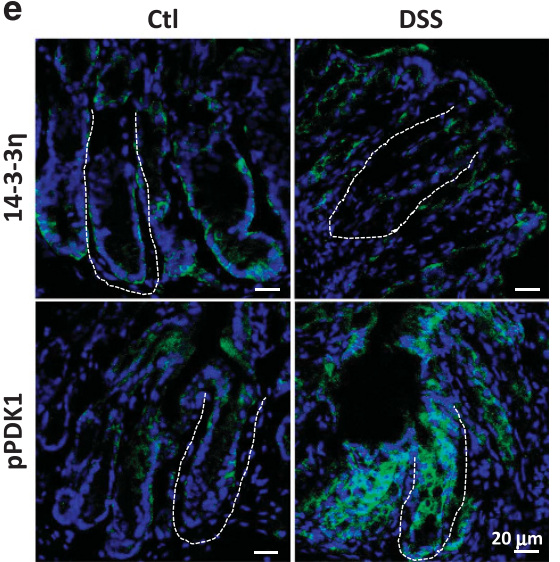

Figure 3 Degradation of 14-3-3 $\eta$ and PDK1 activation are observed during inflammation. (a) pPDK1 and PDK1 were analyzed by western blotting in mucosal samples of C57BL/6J mice exposed to DSS (3\%) for 4 days. Actin was used as a loading control. (b) pPDK1 and PDK1 protein levels were analyzed in SW480 cells transfected with 14-3-30, $14-3-3 \eta, 14-3-3 \beta, 14-3-3 \varepsilon$ or 14-3-3 $\zeta$. Densitometric values for PPDK1 compared with control are displayed. Overexpressed proteins were detected with anti Oct-A (Flag) probe. Actin was used as a loading control. (c) 14-3-3 $\eta, 14-3-3 \theta, 14-3-3 \beta, 14-3-3 \varepsilon$ and 14-3-3 $\zeta$ protein levels were analyzed in the mucosal samples of control and DSS-treated mice (C57BL/6J) by western blotting. Actin was used as a loading control. (d) Graph shows relative densitometric values of pPDK1, 14-3-3 $\eta$ and 14-3-3 $\zeta$ obtained by western blots from mucosal cell lysates of C57BL/6J mice that were treated with DSS. Densitometric values were normalized with respect to controls $(n=5)$. ${ }^{*} P<0.05 ;{ }^{* \star} P<0.001$. (e) pPDK1 (Green) and 14-3-3 $\eta$ (Green) were analyzed in the mucosa of control and colitic mice by immunofluorescence. C57BL/6J received drinking water or drinking water with DSS (3\%) during 4 days. Nuclei, blue. Bar $=20 \mu \mathrm{m}$. Crypt plane is marked by a discontinuous line 
a

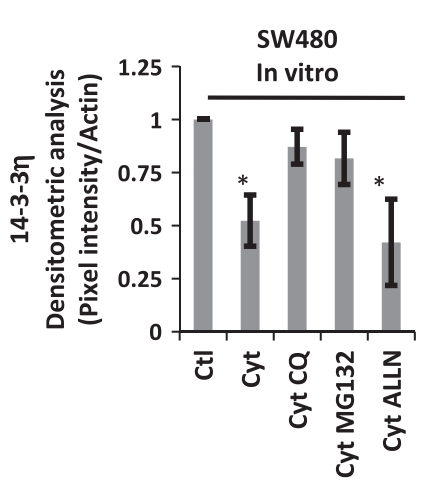

e

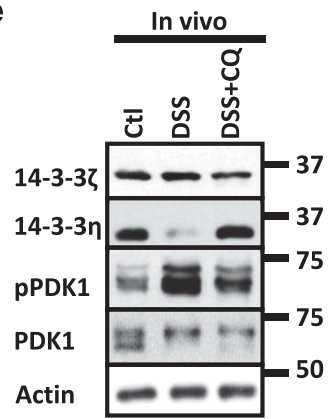

j

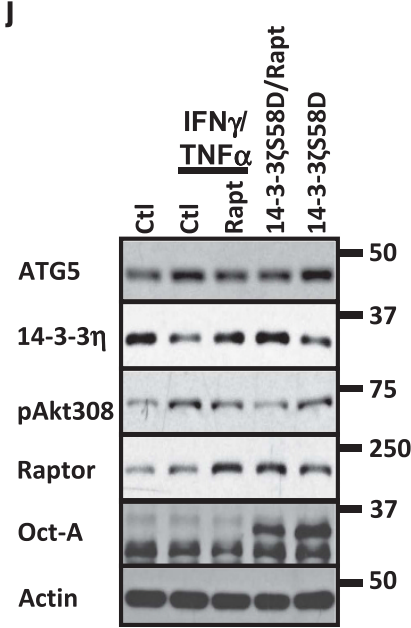

b

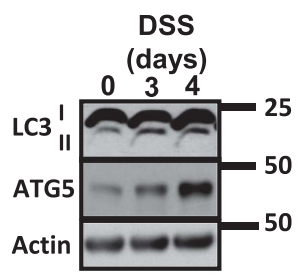

C

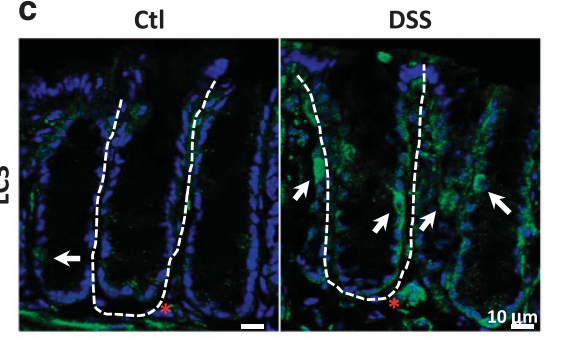

h d

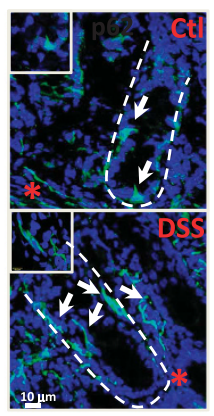

f
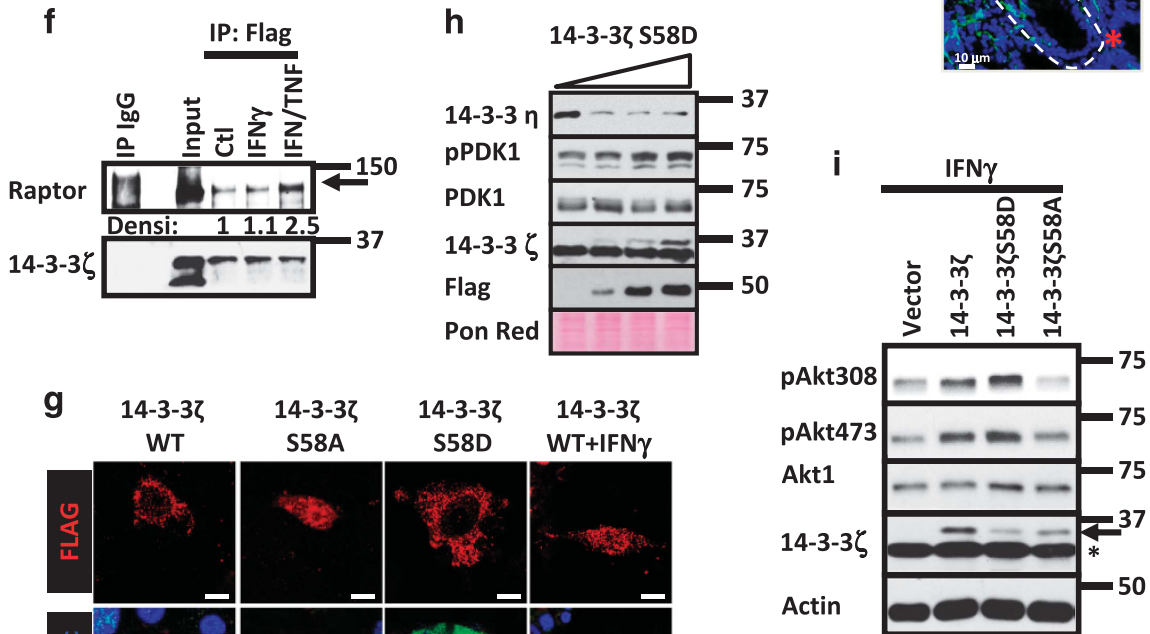

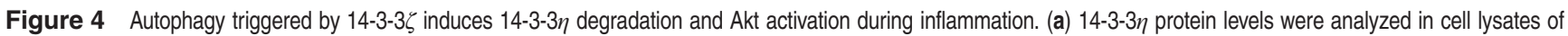
SW480 cells treated with IFN $\gamma / T N F \alpha$ (Cytokines) in the presence or absence of proteasome (MG132), lysosome (CQ) and Calpain (ALLN) inhibitors. Densitometric analysis is shown in graph. $n=3$. ${ }^{*} P<0.05 ;{ }^{* *} P<0.001$. (b) LC3 I-Il and ATG5 were analyzed in mucosal cell lysates of C57BL/6J mice exposed to DSS for $1-4$ days. Actin was used as a loading control. (c) LC3 (green) and (d) p62 (green) localization was analyzed by immunofluorescence in cryosections obtained from colonic mucosa of control mouse and mice exposed to DSS. C57BL/6J received drinking water or drinking water with DSS (3\%) during 4 days. Positive epithelial cells are marked with white arrows. Lamina propria cells that are positive are marked with red star. Crypt axis is marked by white dotted lines. Nuclei are blue. Bar $=10 \mu \mathrm{m}$. (e) 14-3-3 $\zeta, 14-3-3 \eta$, pPDK1 and PDK1 were analyzed in cell lysates of C57BL/6J mice treated for 4 days with DSS (3\%). Mice were injected daily with vehicle alone or with Chloroquine (CQ; $40 \mathrm{mg} / \mathrm{kg}$ ). Actin was used as a loading control.

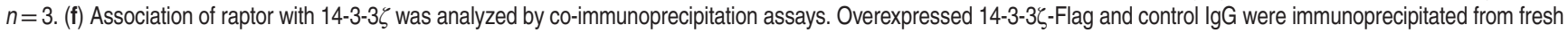
lysates obtained from SW480 control cells or SW480 cells treated with IFN $\gamma$ or IFN $\gamma / T N F \alpha$ for $6 \mathrm{~h}$. Immunoprecipitates were blotted for raptor and 14-3-3

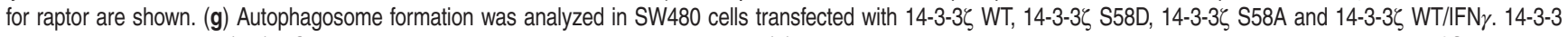

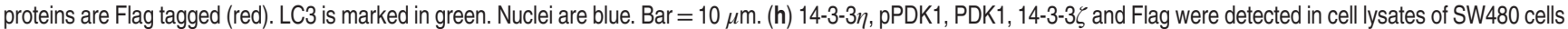

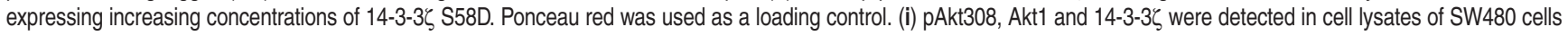

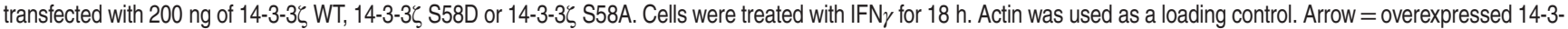

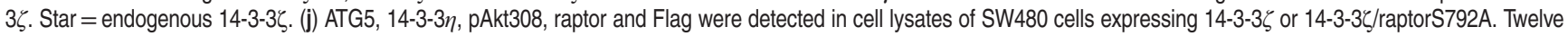

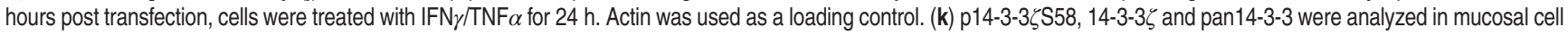
lysates of C57BL/6J mice treated with DSS for 3 days. Actin was used as a loading control 
effect. No changes in PDK1 and 14-3-3 $\zeta$ were detected (Figure 4e). These results suggested that autophagy triggered by inflammatory cytokines was inducing PDK1 activation in IECs at the colonic mucosa.

The mechanism by which inflammation was inducing autophagy in IECs was then assessed. 14-3-37 associates with the regulatory-associated protein of mTOR (Raptor) and inhibits mTORC1 function triggering autophagy. ${ }^{32,33}$ Thus, we investigated whether proinflammatory cytokines could induce the association of 14-3-3 3 with raptor. As shown in Figure 4f, direct association between raptor and 14-3-3 3 was observed in IECs in basal conditions; and this interaction increased in SW480 cells treated with IFN $\gamma / \mathrm{TNF} a$ but not with IFN $\gamma$ alone (Figure 4f). Proinflammatory cytokines enhance phosphorylation of $14-3-3 \zeta$ at the dimeric interface (Supplementary Figure $2 B$ ), thus during inflammation 14-3-3 3 exists in its monomeric

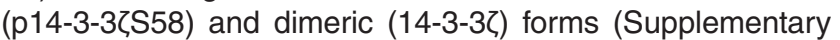
Figure 2C). However, in some conditions p14-3-3\S58 and 14-3-3 $\zeta$ retain its capability to bind its clients but display antagonistic effects in the function of its targets. ${ }^{2}$ For this reason, we investigated the effect of overexpressing 14-3-3ZWT, 14-3-3ZS58D (monomeric form) and 14-3-3ZS58A (dimeric form) ${ }^{34}$ in the accumulation of LC3 in autophagosomes. ${ }^{35}$ As shown in Figure 4g, 14-3-3 358 D but not 14-3-3 WT or 14-3-3 3558 A presence resulted in LC3 accumulation in autophagosomes. Of note, LC3 accumulation was induced by 14-3-3 לWT when IECs were exposed to IFN $\gamma / T N F a$ for $12 \mathrm{~h}$ (Figure $4 \mathrm{~g}$ ). Additionally, the conversion of LC3-I to LC3-II in SW480 cells expressing 14-3-3 mutants confirmed that $14-3-3$ S58D triggers autophagy in IECs; meanwhile, 14-3-3ろS58A has no effect (data not shown). Next, we evaluated the effect of overexpressing

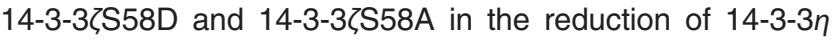
protein levels and in the activation of PDK1. As shown in Figure 4h, increasing concentrations of 14-3-3ZS58D resulted in a decrease in 14-3-3 $\eta$ levels and in PDK1 activation. No changes in PDK1 or endogenous 14-3-3 3 protein levels were detected (Figure 4h). By contrast, no changes in 14-3-3n, PDK1 or 14-3-3 $\zeta$ were observed in SW480 cells expressing 14-3-3ZS58A, but inhibition of PDK1 phosphorylation in a dose-dependent manner was detected (Supplementary Figure 2D). Next, we analyzed the role of 14-3-3 $\zeta$ WT, 14-3-3 $358 \mathrm{D}$ and $14-3-3 \zeta$ S58A in the phosphorylation of Akt at Thr308 and Ser473 in IECs stimulated with IFN $\gamma$ or IFN $\gamma / T N F a$. As shown in Figure $4 \mathrm{i}$, increased presence of pAkt308 and pAkt473 was perceived in IECs transfected with 14-3-3 לWT and 14-3-3לS58D, whereas the phosphorylation of pAkt308 and pAkt473 was reduced in the cells expressing 14-3-3ZS58A. No changes in Akt1 or endogenous 14-3-3 were detected (Figure 4i). Similar effects were noticed in cells treated with IFN $\gamma$ plus TNFa (Supplementary Figure 2E). Moreover, our results also suggested that 14-3-3乙WT and 14-3-3 $358 \mathrm{D}$ enhanced Akt activation downstream of the cytokines, because increased phosphorylation of $\beta$-cat552 was observed in cells transfected with 14-3-3\WT and 14-3-3 3558 when compared with control cells (Supplementary Figure $2 \mathrm{E}$ ). In contrast, $\mathrm{p} \beta$-cat552 presence was reduced in cells expressing 14-3-3 $\zeta$ S58A (Supplementary Figure 2E). No changes in total $\beta$-catenin were seen (Supplementary Figure 2E).
To corroborate that mTORC1 inhibition was responsible for the increase in Akt phosphorylation at Thr308 during inflammation, cell lysates of SW480 cells treated with IFNY alone or with IFNy plus Rapamycin or AktinhibVIII were analyzed for pAkt308. As shown in Supplementary Figure 2F, IFNy increased pAkt308 levels and Rapamycin treatment further enhanced this process but Akt inhibition prevented the increase in pAkt308 induced by IFNy. Inhibition of pAkt308 (AktinhibVIII) and pS6 (Rapamycin) demonstrated the efficiency and specificity of the treatments (Supplementary Figure 2F). Next, we analyzed 14-3-3n, ATG5 and Akt308 levels in cell lysates of SW480 cells that were treated with cytokines after transfection with empty vector or a mutant of raptor that cannot bind to $14-3-3 \zeta^{32}$ As shown in Figure $4 \mathrm{j}$, increased ATG5 accumulation, reduced 14-3-3n protein levels and augmented pAkt308 were seen in control IECs exposed to the cytokines. No changes in raptor were observed (Figure 4j). However, those effects were prevented when the cells treated with the cytokines were transfected with a mutant of raptor that cannot bind to $14-3-3 \zeta .^{32}$ Furthermore, similar to the observed with the cytokines increased ATG5 accumulation, reduced 14-3-3n protein levels and augmented pAkt308 were detected in the monolayers expressing 14-3-3 $3 \mathrm{~S} 58 \mathrm{D}$; however, those changes were reversed when 14-3-3 $3558 D$ was co-transfected with the mutant of raptor (Figure 4j). Of notice, a slight upregulation in raptor was detected in the monolayers expressing 14-3-3̧S58D (Figure 4j). No changes in Akt1 protein levels were detected in any condition (Figure 4j). Taken together, these results suggested that the association of p14-3-3ZS58 with raptor inhibited $\mathrm{mTORC} 1$ and is part of a system that has an important role in the phosphorylation of Akt at Thr308 in IECs.

Next, 14-3-3 and p14-3-3ZS58 levels were analyzed in the mucosa of mice exposed to DSS for 1-4 days to mimic changes at different stages of injury. Densitometric analysis revealed that 14-3-3 , was transiently increased 1-2 days after DSS administration; and its protein levels were back to normal by day 3 (Supplementary Figures $2 \mathrm{G}$ and $4 \mathrm{k}$ ). In contrast, p143-3 З558 oscillated at days 1-3, but was clearly increased by day 4 (Supplementary Figures 2G and $4 \mathrm{j}$ ). No changes were detected in 14-3-3 $\sigma$ another member of the 14-3-3 family of proteins (Supplementary Figure $2 \mathrm{G}$ ), but the results obtained with a pan-14-3-3 antibody suggested that probably other 143-3 family members were also decreased during inflammation (Figure 4k). 14-3-3ろ and p14-3-3ZS58 were seeing in IECs along the crypt axis in control mice as previously reported; ${ }^{2}$ however, after DSS treatment 14-3-3 3 and p14-3-3ZS58 were enriched in IECs located at the crypt base (Supplementary Figure $2 \mathrm{H}$; white arrows). Taken together, these results suggested that 14-3-3 and $p 14-3-3 \zeta S 58$ differentially regulate Akt phosphorylation in IECs during inflammation.

Inhibition of 14-3-3 function during inflammation enhances cell death. Augmented apoptosis (Supplementary Figure $3 \mathrm{~A}-\mathrm{C}$ ) and reduced proliferation in IECs (Supplementary Figures $1 \mathrm{~A}$ and $4 \mathrm{~A}$ ) during colitis are triggered by proinflammatory cytokines, including IFN $\gamma$ (Supplementary Figure 3D). ${ }^{1,36-38}$ Akt1 and 14-3-3Z have an active role in this process. ${ }^{1,2}$ Therefore, we investigated the effect of inhibiting 14-3-3 function in Akt1 signaling using 
a
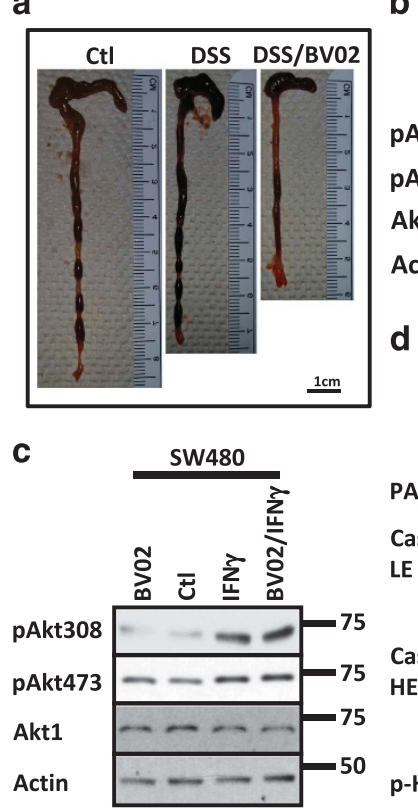

b

d
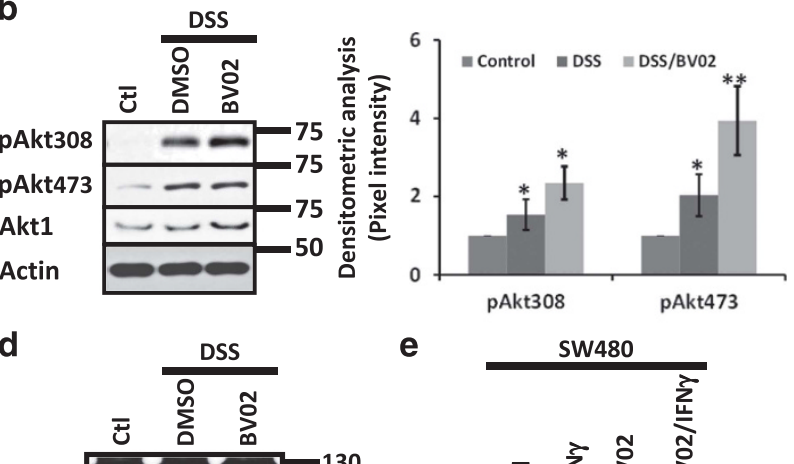

DSS
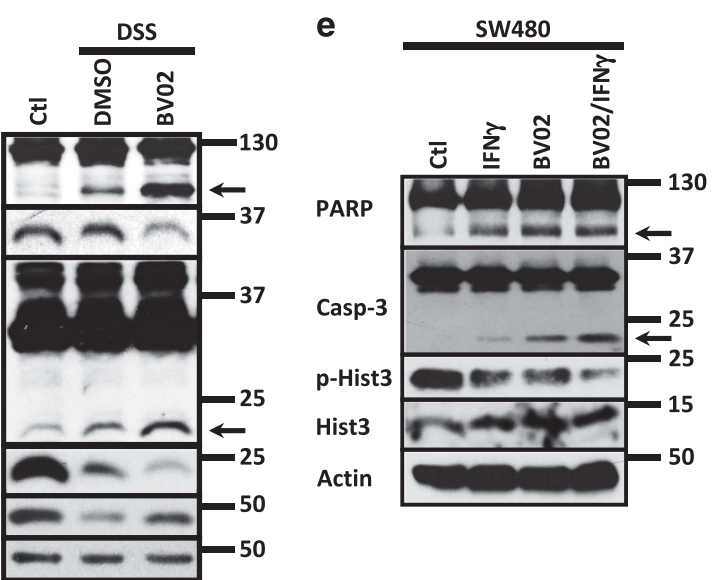

Figure 5 Inhibition of 14-3-3 function enhances Akt activation and triggers apoptosis during inflammation. (a) Representative images of colonic tissue obtained from C57BL/ 6J mice exposed to DSS or DSS/BV02 $(n=8)$. Bar $=1 \mathrm{~cm}$. (b) pAkt308, pAkt473 and Akt1 were analyzed in the mucosa of C57BL/6J mice treated with DSS and DSS/BV02 during 4 days. In all, $10 \mathrm{mg} / \mathrm{kg}$ of BV02 was injected intraperitoneally daily. Actin was used as a loading control. Densitometric analysis of pAkt308 and pAkt473 is presented. $n=7$. ${ }^{*} P<0.05$; ${ }^{* *} P<0.001$. (c) pAkt308, pAkt473 and Akt1 were analyzed in cell lysates of SW480 cells treated with IFN $\gamma$, BV02 (5 $\mu$ M) or IFN $/$ /BV02. Treatment was performed for $36 \mathrm{~h}$. Actin was used as a loading control. $n=3$. (d) PARP and caspase-3 cleavage together with the proliferation markers PCNA and pHist3 were analyzed in the mucosa of mice treated with DSS and DSS/BV02 during 4 days by western blotting. In all, $10 \mathrm{mg} / \mathrm{kg}$ of BV02 was injected intraperitoneally daily. Actin was used as a loading control. $n=3$. (e) PARP and caspase-3 cleavage together with pHist3 and Hist3 protein levels were analyzed in cells exposed to IFN $\gamma$, BV02 or IFN $\gamma / B V 02$. Actin was used as a loading control. $n=3$

the DSS-colitis model. C57BL/6J mice treated with DSS for 4 days were administered daily with the pan-14-3-3 inhibitor BV02 (Co. $10 \mathrm{mg} / \mathrm{kg}$ ) or the vehicle alone (DMSO). DSS treatment reduced colon length when compared with control mice and the treatment with BV02 further enhanced the shortening of the colon (Figure 5a). Moreover, increased phosphorylation of Akt at Ser473 and Thr308 was detected in the mucosa of colitic mice compared with the control (Figure 5b), and the inhibition of 14-3-3 function by BV02 augmented the phosphorylation of both residues (Figure $5 b$ ). Akt1 protein levels were not affected (Figure 5b). p14-3-3 animals, but normal levels were observed in the mucosa of DSS/BV02-treated mice (Supplementary Figure 3E). No changes in 14-3-3 3 were perceived in any of those conditions (Supplementary Figure $3 \mathrm{E}$ ). To corroborate those findings, Akt1, pAkt473 and pAkt308 were analyzed by western blotting in cell lysates of SW480 cells treated with cytokines in the presence or absence of $5 \mu \mathrm{M}$ of BV02. As shown in Figure $5 \mathrm{c}$, IFN $\gamma$ treatment increased Akt phosphorylation at Ser473 and Thr308, and the presence of the inhibitor enhanced Thr308 phosphorylation with minimal or no effect in the phosphorylation of Ser473. Akt1 protein levels remained constant in all conditions (Figure 5c). Taken together, our findings suggested that 14-3-3 function is important for regulating Akt activity in IECs during inflammation.

Active Akt enhances proliferation and inhibits apoptosis. ${ }^{1,2,16}$ Thus, we evaluated apoptosis and proliferation in the mucosa of colitic mice treated with 14-3-3 inhibitor. As shown in Figure $5 \mathrm{~d}$, decreased proliferation (pHist3 and PCNA) and augmented apoptosis (PARP and Casp-3 cleavage) were seen in the mucosa of colitic mice and were increased in the animals treated with DSS plus the 14-3-3 inhibitor (Figure 5d) despite Akt hyperactivation (Figure 5c). Therefore, apoptosis and proliferation were analyzed in SW480 cells treated with IFNY or IFNY plus BV02. IFNY increased apoptosis in IECs (Supplementary Figures 3D and $5 e)$ and inhibition of 14-3-3 function with BV02 $(5 \mu \mathrm{M})$ augmented the cleavage of PARP and Casp-3 (Figure 5e, arrows). 14-3-3 inhibitor also potentiated the inhibition of cell proliferation (pHist3) triggered by the IFNy (Figure 5e). BV02 alone augmented apoptosis and inhibited proliferation in IECs (Figure 5e). Similar effects were observed in RKO cells (data not shown). Taken together, these results demonstrate that 14-3-3 inhibition enhanced Akt activation, augmented apoptosis and decreased cell proliferation in IECs.

Akt induces apoptosis in IECs during inflammation. Akt activation after 14-3-3 inhibition did not prevent cell death (Figure 5); and Akt inhibition reduces colitis symptoms. ${ }^{1}$ 
Thus, we investigated the effect of inhibiting Akt in the development of colitis. As shown in Figure 6a, mice that received DSS plus Akt inhibitor VIII developed substantially milder colitis in comparison with DSS/DMSO-treated animals as indicated by reduced DAl. Moreover, Akt inhibition also resulted in a longer and healthier colon (Figure 6b). Efficiency of the Akt inhibitor was analyzed by western blotting (Figure 6b).

Proinflammatory cytokines induce the accumulation of active Akt at nuclear compartment, ${ }^{2}$ and nuclear Akt can promote cell-cycle progression or apoptosis. ${ }^{39}$ Thus, we speculated that during inflammation nuclear Akt could trigger apoptosis in IECs. To test our hypothesis, we first analyzed the localization of pAkt308 and pAkt473 in the mucosa of colitic mice. As shown in Supplementary Figure $4 A-C$, inflammation induced accumulation of active Akt in the nucleus of IECs, and that was accompanied with a reduction in cell proliferation as shown by the incorporation in EdU (Supplementary Figure 4A) and PCNA expression (Supplementary Figure 4D). Furthermore, Akt inhibition in DSS-treated mice increased PCNA (Supplementary Figure 4D) and decreased casp-3 cleavage (Figure 6c). To further evaluate the contribution of Akt in the induction of cytokine-induced apoptosis, SW480 cells were treated with IFN $\gamma$ and IFN $\gamma$ plus Akt inhibitor VIII for $24 \mathrm{~h}$ (Figure $6 \mathrm{~d}$ ). IFN $\gamma$ treatment augmented PARP and Caspase-3 cleavage and conversely, the presence of the inhibitor partially reversed this process demonstrating that active Akt has a role in the induction of apoptosis in IECs during inflammation (Figure $6 \mathrm{~d}$ ). Of note, in agreement with the role of Akt1 as a pro-survival molecule, augmented cleavage of PARP and Caspase-3 was observed in IECs exposed to the Akt inhibitor alone (Figure 6d). Decreased Akt Thr308 and Ser473 was observed in cells treated with the Akt inhibitor VIII (Figure 6d). To investigate whether nuclear Akt was inducing cell death during inflammation, we took advantage of gain function approach. Therefore, apoptosis (PARP cleavage) was analyzed in SW480 cells expressing increasing concentrations of Akt1 WT, this approach results in the accumulation of active Akt1 at the nuclear compartment. ${ }^{2}$ As shown in Figure $6 \mathrm{e}$, in BSA-treated monolayers PARP cleavage was only observed when high amounts of Akt1 were transfected. However, in IECs exposed to IFN $\gamma$, Akt1 overexpression triggered apoptosis even with low concentrations (Figure 6e). To investigate whether nuclear Akt was in fact augmenting IEC death during inflammation, we next evaluated the effect of expressing a membrane-bound form of Akt1 that is constitutively active (Akt1.myr), in the induction of apoptosis triggered by IFN $\gamma$. As shown in Supplementary Figure 4E, IFN $\gamma$ treatment augmented PARP cleavage, but this effect was partially prevented when SW480 cells were transfected with Akt1.myr, suggesting that translocation of Akt to the nuclear compartment is important for IEC apoptosis during inflammation. Because BV02 treatment augmented IEC death during colitis (Figure 5d), we then analyzed the cellular distribution of active Akt in control, DSS and DSS/BV02-treated mice. As shown in Figure 6f, high levels of pAkt308, pAkt473 and Akt1 were detected in nuclear extracts obtained from mucosal samples of colitic mice when compared with healthy animals. Moreover, a further increase in those molecules was detected when DSS-treated animals were injected with the pan-14-3-3 inhibitor BV02 (Figure 6f). Thus, all these results suggest that nuclear accumulation of Akt1 during inflammation triggers apoptosis in IECs.

On the basis of these results, we speculated that 14-3-3n could be a part of a mechanism that inhibits PDK1 function during inflammation to tamper Akt activation in order to prevent death in IECs. Therefore, we analyzed pPDK1 presence and apoptosis induction after cytokine treatment in IECs overexpressing 14-3-3n. To this end, IECs transfected with increasing concentrations of 14-3-3 or empty vector were treated with cytokines for $24 \mathrm{~h}$. PDK1, pPDK1, 14-3-3द, 14-3-3n, cleaved caspase-3 and PARP were analyzed by western blotting. As shown in Figure 7a, Casp-3 and PARP cleavage was detected in control IECs treated with cytokines; however, this phenomenon was prevented in cells expressing 14-3-3n. Furthermore, the phosphorylation of PDK1 after cytokine treatment observed in control monolayers was also prevented when 14-3-3 $\eta$ was overexpressed (Figure 7a). 14-3-3n Overexpression did not affect in 14-3-3Z or PDK1 (Figure 7a). Finally, we analyzed the role of PDK1 on Akt1 localization. Therefore, SW480 cells expressing Akt1-GFP were treated with cytokine or cytokine plus PDK1 inhibitor. As shown in Figure 7b, cytokine treatment induced Akt1 nuclear accumulation in IECs, but this effect was prevented when cells were pretreated with the PDK1 inhibitor GSK23334470. Moreover, PDK1 inhibition or 14-3-3n overexpression reduced cytokine-mediated IEC death to a similar extent (Figure 7c) and also prevented the cleavage of caspase-3 triggered by the cytokine treatment (Figure 7d). Thus, all together these results demonstrate that downregulation of 14-3-3n during inflammation results in activation of PDK1, Akt1 activation and likely leads to Akt-induced cell death.

\section{Discussion}

Changes in IEC homeostasis due to proinflammatory intermediaries are a hallmark of inflammatory bowel diseases. Cytokines such as IFN $\gamma$ and TNFa are critical mediators that contribute to the epithelial barrier breakdown by disassembling tight junctions, reducing the rate of intestinal epithelial cell migration, decreasing IEC proliferation and increasing cell death. ${ }^{1,2,40,41}$ Cytokine mediators regulate biological processes by altering several signaling pathways including Akt, NOs and JAK/STAT.,42,43 However, the mechanisms underlying those processes are incompletely understood.

In this report, we showed that proinflammatory cytokines enhance Akt activation in IECs during inflammation. Using complementary in vivo and in vitro approaches, we show that proinflammatory cytokines stimulate Akt Thr308 phosphorylation in IECs by inducing degradation of the PDK1 inhibitor, 14$3-3 \eta$, through a process that involves inhibition of $\mathrm{mTORC} 1$ by 14-3-3 $\zeta$ and likely autophagy. Consistent with our findings, Akt Thr308 phosphorylation has been observed in other models where raptor has been downregulated or inactivated (rapamycin). ${ }^{44-46}$ Thus, based on this premise, we suggest that probably association of raptor with 14-3-3 proteins could serve two major purposes: (1) inhibit mTORC1 function to trigger autophagy and therefore 14-3-3n degradation and PDK1 activation; and (2) release the TOR components to 
a

b
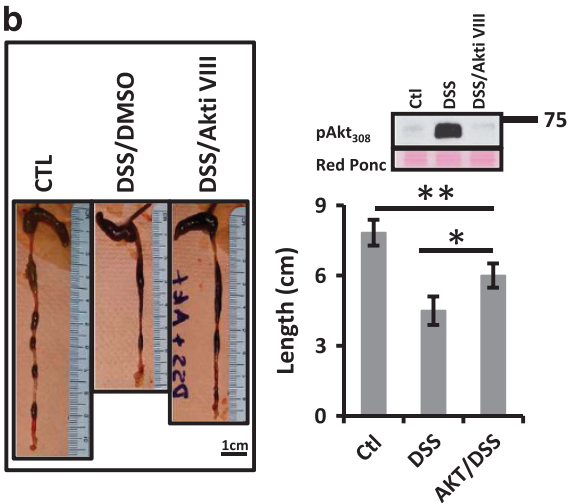

C

DSS
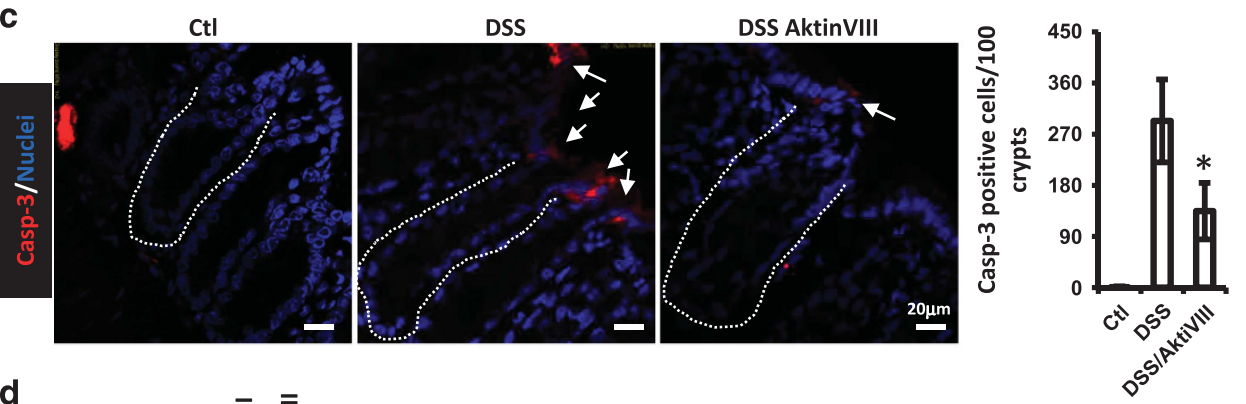

d

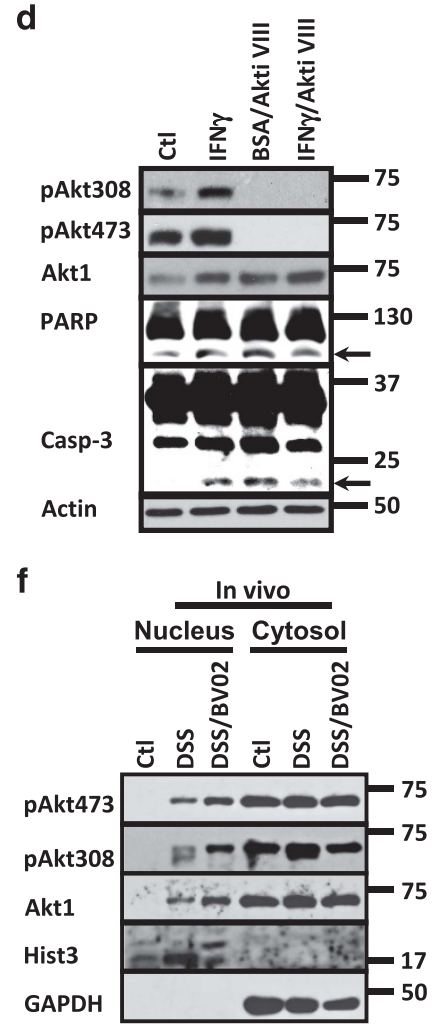

e

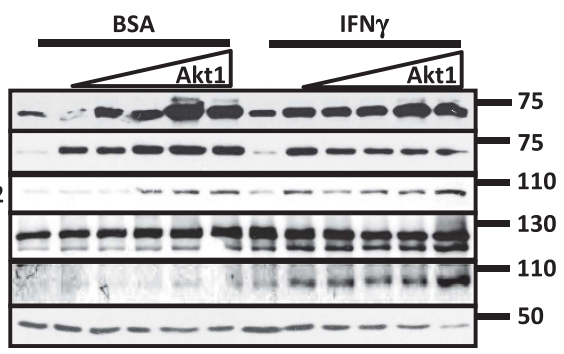

Actin

\section{Akt1}

pAkt308

$\mathrm{p} \beta$-cat552

PARP HE

PARPC LE

Figure 6 Akt inhibition reduces death in IECs during inflammation. (a) DSS-induced cumulative disease activity index-a cumulative disease activity index was calculated using clinical symptom data for each time point for DMSO $(\square)$ and Akt inhibitor VIII ( $\downarrow)$ treated mice. ${ }^{*} P<0.05 .{ }^{* *} P<0.001$. (b) Representative images obtained from colons of C57BL/6J mice exposed to water, DSS/DMSO and DSS/Akt inhibitor VIII. Quantification is shown in the graph. In all, $10 \mathrm{mg} / \mathrm{kg}$ of weight of Akt inhibitor VIII was administered daily via peritoneum. Akt pAkt308 protein levels in mucosal cell lysates are shown. ${ }^{*} P<0.05,{ }^{* *} P<0.001 . n=5$. Bar $=1 \mathrm{~cm}$. (c) Immunofluorescence staining for active caspase-3 (red) in the mucosa of C57BL/6J mice treated with water, DSS/DMSO and DSS/Akt inhibitor VIII. Caspase-3-positive cells are marked by white arrows. Crypt axis is marked by white dotted lines. $\mathrm{Bar}=20 \mu \mathrm{m}$. Quantification is shown in graph. ${ }^{*} P<0.05 . n=3$. (d) pAkt308, pAkt473, Akt1, PARP and Caspase-3 were analyzed in cell lysates of SW480 cells treated with IFN $\gamma$, Akt Inhibitor VIII (2.12 $\mu \mathrm{M})$ or IFN $\gamma /$ Akt Inhibitor VIII. Treatment was performed for $36 \mathrm{~h}$. Actin was used as a loading control. $n=3$. (e) Akt1, pAkt308, p $\beta$-Cat552 and PARP were analyzed in cell lysates of SW480 expressing increasing concentrations of hAkt1 flag tagged. Cells were treated with BSA or IFN $\gamma$ during $18 \mathrm{~h}$. Actin was used as a loading control. $n=3$. (f) pAkt473, pAkt308 and Akt1 were analyzed in nuclear fractions of IECs obtained from mice exposed to water, DSS or DSS/BV02 for 4 days. In all, $10 \mathrm{mg} / \mathrm{kg}$ of BV02 was injected intraperitoneally daily. Mice were injected Histone-3 that was used as a marker for nuclear fraction. GAPDH was used as a marker for cytosolic fractions. $n=3$ 
a

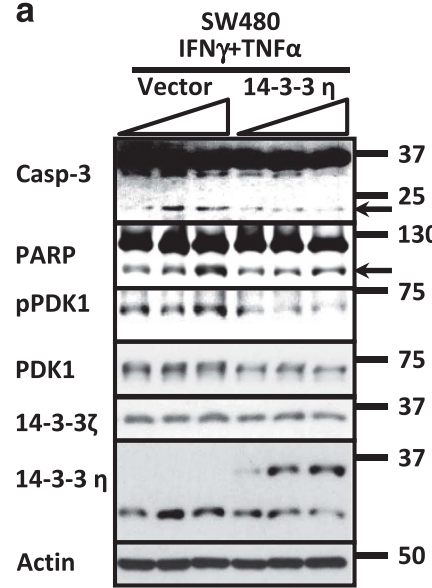

b

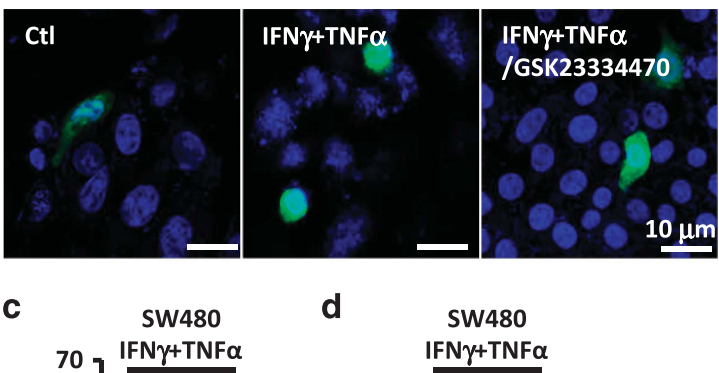

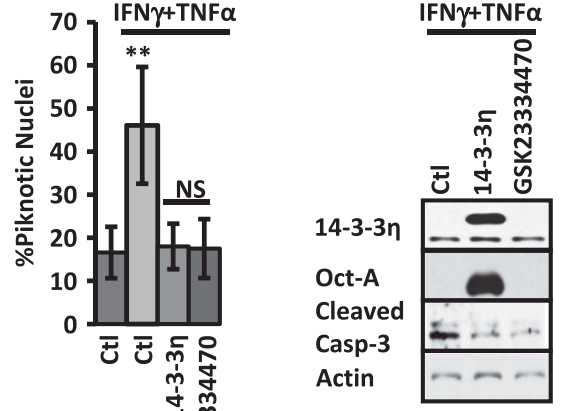

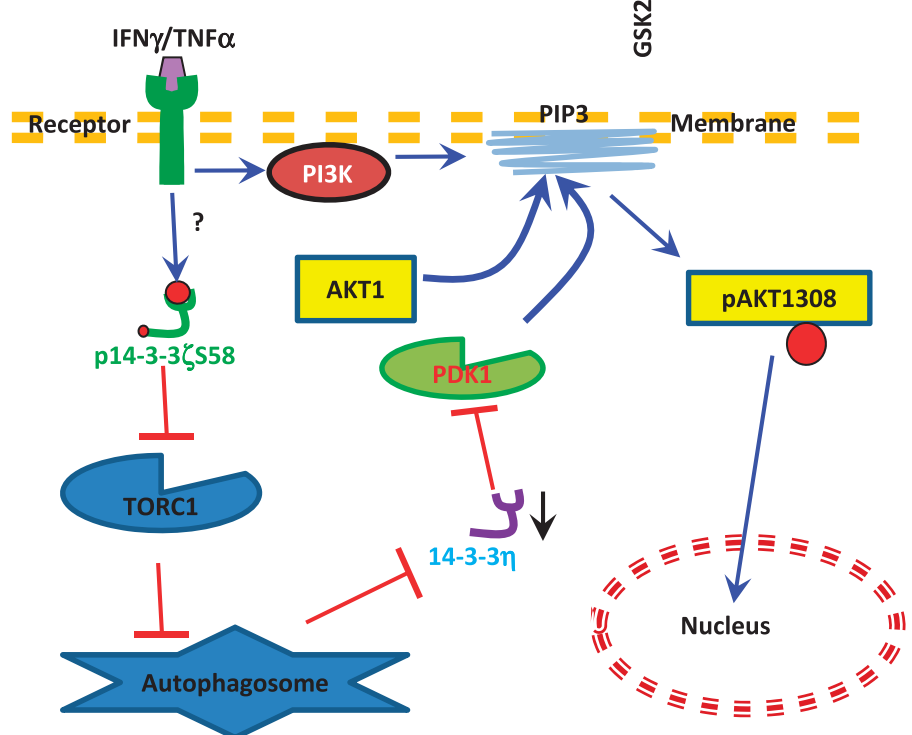

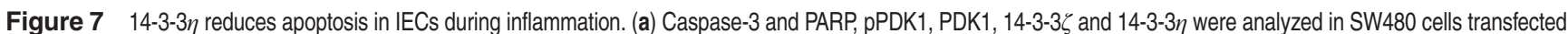
with increasing concentrations of 14-3-37. IECs were exposed to IFN $\gamma / T N F \alpha$ for $24 \mathrm{~h}$. Arrows mark cleaved caspase-3 and PARP. Actin was used as a loading control. (b) SW480 transfected with Akt1-GFP was stimulated with cytokines for $24 \mathrm{~h}$ in the presence or absence of the PDK1 inhibitor (GSK23334470; $50 \mu \mathrm{M}$ ). The inhibitor was added 30 min before cytokine treatment. Nuclei $=$ Blue. Bar $=10 \mu \mathrm{m}$. (c) Nuclear piknosis and (d) 14-3-3 $\eta$, Oct-A(Flag) and caspase-3 cleavage were analyzed in SW480 cells that were transfected with 14-3-3 $(2 \mu \mathrm{g})$ or treated with PDK1 inhibitor (GSK23334470; $50 \mu \mathrm{M}$ ), before exposure to IFN $\gamma / \mathrm{TNF} \alpha$ for $24 \mathrm{~h}$. Actin was used as a loading control. ${ }^{* *} P<0.001$. $n=3$. (e) Hypothetical model for Akt activation by IFN $\gamma$ and 14-3-3 proteins in IECs

allow mTORC2 assembly. The final output of these processes could lead to the phosphorylation of Akt at two key residues, Ser473 and Thr308, which could result in Akt full activation as shown by Sarbassov et al. ${ }^{11}$

Contrary to the previous reports where mTORC1 was inactivated by exogenous means, here we shown that mTORC1 and Akt may be intrinsically modulated by 14-3-3 proteins. Specifically, our findings suggest (Figure 7c) that cytokine stimulation triggered the phosphorylation of 14-3-3 $\zeta$ and its association with raptor. The association of p14-3-3 לS58 with raptor could prevent the binding of raptor with the TOR complex consequently inhibiting mTORC1 activity and triggering autophagy. ${ }^{32}$ This process likely results in 14-3-3n degradation via autophagolysosomes; therefore, in some conditions 14-3-3 $\zeta$ could control Akt full activation and its biological functions. We believe that during inflammation 14-3-3 function/presence could be important to mediate the balance between the activities of Akt and the mTOR complexes, which is important to inhibit cell death. For instance, it could act as a switch that controls a major cellular energy consumer, mTORC1, and a major energy producer, Akt. But also could have an important role to inhibit autophagy 
mTORC1 mediated, ${ }^{47-50}$ which is an important process to prevent autophagy-mediated cell death. ${ }^{47,51}$

Cytokine-driven autophagy has been observed in several pathological conditions including atherosclerosis, ${ }^{52}$ myopathy ${ }^{53}$ and arthritis, ${ }^{54}$ which has been linked to TNF $a$ and IFN $\gamma$ presence. But, contrary to those reports where autophagy acts as a suicidal mechanism, ${ }^{55,56}$ our results suggested that the major purpose of the accompanying increase in autophagy observed during inflammation is to fully activate Akt1, a major antiapoptotic signaling molecule. ${ }^{16}$ However, the continuous cytokine stimulation of the inflamed mucosa ${ }^{57,58}$ might result in the dysregulation of the machinery that controls Akt signaling pathway, leading to uncontrolled Akt activation and cell death. This is a plausible scenario because proinflammatory cytokines can also affect other biological processes that control Akt function such as protein synthesis ${ }^{2}$ and/or Akt targeting/shuttling, ${ }^{2}$ which is known to be important for controlling Akt destiny and function. ${ }^{39}$

14-3-3 $\zeta$ stimulates PI3K signaling ${ }^{59}$ and also activates PDK1, thus its presence could be important but not essential $^{60}$ to induce Akt activation, those properties in 14-3-3 $\zeta$ could explain in part the tumor-promoting function attributed to $14-3-3 \zeta$ upregulation. ${ }^{61}$ Additionally, the ratio maintained between the dimeric and monomeric forms of 14-3-3 3 might have a role in controlling Akt activity; and it is possible that oscillations in the amount of p14-3-3 3558 are necessary not only to maintain the Akt signal but also to control its cellular localization, which is important because Akt phosphorylates at least 100 non-redundant substrates in different cell compartments. ${ }^{62}$ In this context, we now show that during inflammation the monomeric form of 14-3-37 augments and active Akt increases in the nucleus where triggers IEC death. These findings are not the first one to suggest a role for Akt in apoptosis induction; as prolonged Akt activation has been shown to trigger apoptosis, ${ }^{1,63}$ and apoptotic stimuli have been shown to induce Akt accumulation in the nucleus, ${ }^{39}$ but here we observed that proinflammatory cytokines augmented the pro-apoptotical function of Akt by enhancing its full activation and nuclear accumulation through affecting the function of 14-3-3 proteins. Therefore, we speculate that the therapies using Akt inhibitors may be useful to reduce apoptosis in IECs of patients with IBD, but the complex interplay between the cellular distribution and temporal activation of Akt has to be taken into account when these treatments are designed. In fact, lack of understanding of these processes could explain why therapies aimed merely to inhibit Akt activation are moderately successful in experimental cancer therapy ${ }^{64,65}$ and in preventing IEC cell death during inflammation as shown here.

Finally, given that the vast number of clients of the 14-3-3 proteins is not difficult to envision that similar mechanisms could be used by these proteins to control other signaling pathways, during biological or pathological processes. Thus, understanding how 14-3-3 proteins can inhibit or activate other signaling molecules would be an important step in the process of create new treatments aimed to regulate biological functions through controlling 14-3-3 molecules. In conclusion, in this work we highlight a new mechanism by which 14-3-3 proteins can tamper Akt activation in IECs in order to control proliferation and apoptosis during inflammation.

\section{Materials and Methods}

Antibodies and reagents. Recombinant human IFN $\gamma$ and $\mathrm{TNF} \alpha$ were obtained from Peprotech (Rocky Hill, NJ, USA), and used at a concentration of 100 and $110 \mathrm{U} / \mathrm{ml}$ for in vitro treatment, respectively. $\mathrm{p}-14-3-3 \zeta$ antibody was obtained from Santa Cruz (CA, USA; Cat. No. sc-101623). Information about other primary antibodies and plasmids was previously reported. ${ }^{2}$ Plasmids were transfected with Lipofectamine 2000 (Invitrogen, Grand Island, NY, USA). Akt inhibitor VIII (Calbiochem, Darmstadt, Germany), PDK1 inhibitor GSK2334470 (Sigma, St. Louis, MO, USA), Rapamycin (Calbiochem) and pan14-3-3 inhibitor (Sigma) were used according to the supplier's recommendations. Dextran Sulfate Sodium Salt (40-50 K, MW) was purchased from Affymetrix, USB products (Santa Clara, CA, USA). Secondary antibodies were purchased from Invitrogen and Jackson ImmunoResearch (West Grove, PA, USA).

14-3-3Z-GFP generation. GFP-tagged human tyrosine 3-monooxygenase/

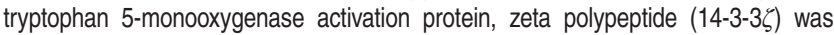
generated by cloning. Full-length $14-3-3 \zeta$ CDS was amplified from pCMV6-EntryYWHAZ cDNA ORF clone (Origene, Rockville, MD, USA) using the primers $F$ $5^{\prime}$-gcg agc tca tgg ata aaa atg a-3' and R $5^{\prime}$-tac ccg ggc att ttc ccc tcc tt-3' and cloned into the Sacl and Xmal sites of the expression vector pEGFP-C1 (Clontech, Mountain View, CA, USA). The resulting plasmid was verified by DNA sequencing.

Cell culture. Intestinal epithelial cell lines SW480 and RKO were obtained from ATCC grown in DMEM with 10\% fetal calf serum and antibiotics. Cells were maintained in a humidified incubator with $5 \% \mathrm{CO}_{2}$. For functional studies, cells were seeded onto collagen-coated, permeable filters (Costar, Corning, NY, USA) or glass coverslips.

Animal experiments. All procedures with animals were reviewed and approved by the CINVESTAV Institutional Committee for Care and Use of Laboratory Animals (CICUAL) and were performed according to CONACYT criteria. Male C57BI/6J mice (7-8 weeks old) were obtained from The Jackson Laboratories (Bar Harbor, ME, USA). Animals were housed in a standard day and night cycle, with free access to food and water and experiments performed as previously reported by us. ${ }^{19}$ Randomization but not blinding protocols were applied to all experiments with animals. Sample size was defined using pre-established protocols. ${ }^{66}$ Proteins were analyzed by western blotting and by indirect immunofluorescence staining as previously described. ${ }^{19}$

Western blot assays. Samples were collected in Ripa lysis buffer (150 mM $\mathrm{NaCl}, 1 \% \mathrm{NP}-40,0.5 \%$ deoxycholic acid, $0.1 \%$ SDS, $50 \mathrm{mM}$ Tris [pH 8.0]) supplemented with protease and phosphatase inhibitors (Sigma), sonicated and cleared by centrifugation. Protein concentration was determined by BCA (Pierce, Rockford, IL, USA), and samples boiled in SDS sample buffer with $50 \mathrm{mM}$ dithiothreitol (Sigma). Equal amounts of protein were separated by SDS-PAGE and transferred onto nitrocellulose membranes. Membranes were blocked for $1 \mathrm{~h}$ with $2 \%$ wt/vol dry milk or BSA (Sigma) in Tris-buffered (Sigma) saline containing $0.1 \%$ Tween-20 (Sigma) and incubated with primary antibodies in blocking buffer overnight at $4^{\circ} \mathrm{C}$.

Immunofluorescence microscopy. Tissue sections were fixed with $4 \%$ PFA (15 min; Sigma), and then permeabilized with $100 \%$ methanol $\left(20 \mathrm{~min},-20^{\circ}\right.$ C; Sigma). Samples were blocked with $2 \% \mathrm{wt} / \mathrm{vol}$ BSA (Sigma) for $1 \mathrm{~h}$ and incubated with primary antibodies overnight at $4{ }^{\circ} \mathrm{C}$. After incubation with fluorophore-labeled secondary antibodies for $1 \mathrm{~h}$, nuclei were stained with ToPro3 iodide (Molecular Probes, Grand Island, NY, USA), and coverslips were mounted in p-phenylene. Images were taken on an LSM 710 confocal microscope (Zeiss, Jena, Germany) with Plan-NEOFLUAR 1003/1.3 oil, 403/1.3 oil and 203/0.5 dry objectives, with software supplied by the vendor.

Cellular fractionation. Cytoplasmic and nuclear extracts were prepared using the NE-PER Nuclear and Cytoplasmic Extraction Kit (Thermo Scientific, Waltham, MA, USA) according to the manufacturer's protocol.

Histological analysis. Nuclear piknosis was analyzed as previous reported by us. ${ }^{67}$

Statistics. Dunnett's post-test after one-way ANOVA or two-tailed Student's $t$-test was used to analyze the data (GraphPad Software, La Jolla, CA, USA). $P<0.05$ was considered as statistically significant. 


\section{Conflict of Interest}

The authors declare no conflict of interest.

Acknowledgements. This study was supported by grants from the American Gastroenterological Association (Research Scholar Award to PN), the Crohn's and Colitis Foundation of America (Career Development Award to PN), Conacyt (175854 to PND), the Miguel Alemán Valdez foundation (Clinical Research award to PND), the Glaxo-SmithKline International Award from the Japanese Society of Immunology and Allergology in Otolaryngology (to RK) and in part by grants from the $\mathrm{NIH}$ (DK072564, DK061379, DK079392 to CAP; and DK055679 and DK059888 to AN). We acknowledge the technical assistance of QFB. Leticia Alemán Lazarini in the Confocal Microscope Facility at CINVESTAV.

1. Nava $P$, Koch S, Laukoetter MG, Lee WY, Kolegraff K, Capaldo CT et al. Interferon-gamma regulates intestinal epithelial homeostasis through converging beta-catenin signaling pathways. Immunity 2010; 32: 392-402.

2. Nava P, Kamekura R, Quiros M, Medina-Contreras O, Hamilton RW, Kolegraff KN et al. IFNgamma-induced suppression of beta-catenin signaling: evidence for roles of Akt and 14.3.3zeta. Mol Biol Cell 2014; 25: 2894-2904.

3. Gardino AK, Yaffe MB. 14-3-3 proteins as signaling integration points for cell cycle control and apoptosis. Semin Cell Dev Biol 2011; 22: 688-695.

4. Hermeking H. The 14-3-3 cancer connection. Nat Rev Cancer 2003; 3: 931-943.

5. Downward J. PI 3-kinase, Akt and cell survival. Semin Cell Dev Biol 2004; 15: 177-182.

6. Xu N, Lao Y, Zhang Y, Gillespie DA. Akt: a double-edged sword in cell proliferation and genome stability. J Oncol 2012; 2012: 951724.

7. Mora A, Komander D, van Aalten DM, Alessi DR. PDK1, the master regulator of AGC kinase signal transduction. Semin Cell Dev Biol 2004; 15: 161-170.

8. Fujita N, Sato S, Ishida A, Tsuruo T. Involvement of Hsp90 in signaling and stability of 3-phosphoinositide-dependent kinase-1. J Biol Chem 2002; 277: 10346-10353.

9. Frodin M, Jensen CJ, Merienne K, Gammeltoft S. A phosphoserine-regulated docking site in the protein kinase RSK2 that recruits and activates PDK1. EMBO J 2000; 19: 2924-2934.

10. Sato S, Fujita N, Tsuruo T. Regulation of kinase activity of 3-phosphoinositide-dependent protein kinase-1 by binding to 14-3-3. J Biol Chem 2002; 277: 39360-39367.

11. Sarbassov DD, Guertin DA, Ali SM, Sabatini DM. Phosphorylation and regulation of Akt/PKB by the rictor-mTOR complex. Science 2005; 307: 1098-1101.

12. Delcommenne M, Tan C, Gray V, Rue L, Woodgett J, Dedhar S. Phosphoinositide-3-OH kinase-dependent regulation of glycogen synthase kinase 3 and protein kinase B/AKT by the integrin-linked kinase. Proc Natl Acad Sci USA 1998; 95: 11211-11216.

13. Kawakami $\mathrm{Y}$, Nishimoto $\mathrm{H}$, Kitaura J, Maeda-Yamamoto M, Kato RM, Littman DR et al. Protein kinase $C$ betall regulates Akt phosphorylation on Ser- 473 in a cell type- and stimulusspecific fashion. J Biol Chem 2004; 279: 47720-47725.

14. Yang ZZ, Tschopp O, Hemmings-Mieszczak M, Feng J, Brodbeck D, Perentes E et al. Protein kinase B alpha/Akt1 regulates placental development and fetal growth. J Biol Chem 2003; 278: 32124-32131.

15. Vanhaesebroeck B, Alessi DR. The PI3K-PDK1 connection: more than just a road to PKB. Biochem J 2000; 346(Pt 3): 561-576.

16. Brazil DP, Yang ZZ, Hemmings BA. Advances in protein kinase B signalling: AKTion on multiple fronts. Trends Biochem Sci 2004; 29: 233-242.

17. Toker A, Yoeli-Lerner M. Akt signaling and cancer: surviving but not moving on. Cancer Res 2006; 66: 3963-3966.

18. Perse M, Cerar A. Dextran sodium sulphate colitis mouse model: traps and tricks. J Biomed Biotechnol 2012; 2012: 718617.

19. Laukoetter MG, Nava P, Lee WY, Severson EA, Capaldo CT, Babbin BA et al. JAM-A regulates permeability and inflammation in the intestine in vivo. $J$ Exp Med 2007; 204: 3067-3076.

20. Fang D, Hawke D, Zheng Y, Xia Y, Meisenhelder J, Nika $\mathrm{H}$ et al. Phosphorylation of betacatenin by AKT promotes beta-catenin transcriptional activity. J Biol Chem 2007; 282: $11221-11229$.

21. Jope RS, Yuskaitis CJ, Beurel E. Glycogen synthase kinase-3 (GSK3): inflammation, diseases, and therapeutics. Neurochem Res 2007; 32: 577-595.

22. Shaw J, Kirshenbaum LA. Prime time for JNK-mediated Akt reactivation in hypoxiareoxygenation. Circ Res 2006; 98: 7-9.

23. Li X, Lu Y, Jin W, Liang K, Mills GB, Fan Z. Autophosphorylation of Akt at threonine 72 and serine 246. A potential mechanism of regulation of Akt kinase activity. J Biol Chem 2006; 281: 13837-13843.

24. Breuleux M, Klopfenstein M, Stephan C, Doughty CA, Barys L, Maira SM et al. Increased AKT S473 phosphorylation after mTORC1 inhibition is rictor dependent and does not predict tumor cell response to PI3K/mTOR inhibition. Mol Cancer Ther 2009; 8: 742-753.

25. Guertin DA, Stevens DM, Saitoh M, Kinkel S, Crosby K, Sheen JH et al. mTOR complex 2 is required for the development of prostate cancer induced by Pten loss in mice. Cancer Cell 2009; 15: 148-159.
26. Casamayor A, Morrice NA, Alessi DR. Phosphorylation of Ser-241 is essential for the activity of 3-phosphoinositide-dependent protein kinase-1: identification of five sites of phosphorylation in vivo. Biochem J 1999; 342(Pt 2): 287-292.

27. Lilienbaum A. Relationship between the proteasomal system and autophagy. Int J Biochem Mol Biol 2013; 4: 1-26.

28. Assani K, Tazi MF, Amer AO, Kopp BT. IFN-gamma stimulates autophagy-mediated clearance of Burkholderia cenocepacia in human cystic fibrosis macrophages. PLOS ONE 2014; 9: e96681.

29. Fougeray S, Mami I, Bertho G, Beaune P, Thervet E, Pallet N. Tryptophan depletion and the kinase GCN2 mediate IFN-gamma-induced autophagy. J Immunol 2012; 189: 2954-2964.

30. Pyo JO, Yoo SM, Ahn HH, Nah J, Hong SH, Kam Tl et al. Overexpression of Atg5 in mice activates autophagy and extends lifespan. Nat Commun 2013; 4: 2300.

31. Bjorkoy G, Lamark T, Pankiv S, Overvatn A, Brech A, Johansen T. Monitoring autophagic degradation of p62/SQSTM1. Methods Enzymol 2009; 452: 181-197.

32. Gwinn DM, Shackelford DB, Egan DF, Mihaylova MM, Mery A, Vasquez DS et al. AMPK phosphorylation of raptor mediates a metabolic checkpoint. Mol Cell 2008; 30: 214-226.

33. Pozuelo-Rubio M. 14-3-3 proteins are regulators of autophagy. Cells 2012; 1: 754-773.

34. Woodcock JM, Murphy J, Stomski FC, Berndt MC, Lopez AF. The dimeric versus monomeric status of 14-3-3zeta is controlled by phosphorylation of Ser58 at the dimer interface. J Biol Chem 2003; 278: 36323-36327.

35. Kabeya Y, Mizushima N, Yamamoto A, Oshitani-Okamoto S, Ohsumi Y, Yoshimori T. LC3, GABARAP and GATE16 localize to autophagosomal membrane depending on form-II formation. J Cell Sci 2004; 117(Pt 13): 2805-2812.

36. Boehm U, Klamp T, Groot M, Howard JC. Cellular responses to interferon-gamma. Annu Rev Immunol 1997; 15: 749-795.

37. Fish SM, Proujansky R, Reenstra WW. Synergistic effects of interferon gamma and tumour necrosis factor alpha on T84 cell function. Gut 1999; 45: 191-198.

38. Gibson PR. Apoptosis or necrosis-colonic epithelial cell survival. Novartis Found Symp 2004; 263: 133-145; discussion 145-150, 211-138.

39. Maddika S, Ande SR, Wiechec E, Hansen LL, Wesselborg S, Los M. Akt-mediated phosphorylation of CDK2 regulates its dual role in cell cycle progression and apoptosis. J Cell Sci 2008; 121(Pt 7): 979-988.

40. Gunzel D, Florian P, Richter JF, Troeger H, Schulzke JD, Fromm M et al. Restitution of single-cell defects in the mouse colon epithelium differs from that of cultured cells. Am J Physiol Regul Integr Comp Physiol 2006; 290: R1496-R1507.

41. Tong $Q$, Vassilieva EV, Ivanov Al, Wang Z, Brown GT, Parkos CA et al. Interferon-gamma inhibits T84 epithelial cell migration by redirecting transcytosis of beta1 integrin from the migrating leading edge. J Immunol 2005; 175: 4030-4038.

42. Nguyen H, Ramana CV, Bayes J, Stark GR. Roles of phosphatidylinositol 3-kinase in interferon-gamma-dependent phosphorylation of STAT1 on serine 727 and activation of gene expression. J Biol Chem 2001; 276: 33361-33368.

43. Frost RA, Nystrom GJ, Lang $\mathrm{CH}$. Endotoxin and interferon-gamma inhibit translation in skeletal muscle cells by stimulating nitric oxide synthase activity. Shock 2009; 32: 416-426.

44. Chen XG, Liu F, Song XF, Wang ZH, Dong ZQ, Hu ZQ et al. Rapamycin regulates Akt and ERK phosphorylation through mTORC1 and mTORC2 signaling pathways. Mol Carcinog 2010; 49: 603-610.

45. Wan X, Harkavy B, Shen N, Grohar P, Helman LJ. Rapamycin induces feedback activation of Akt signaling through an IGF-1 R-dependent mechanism. Oncogene 2007; 26: 1932-1940

46. Wang S, Amato KR, Song W, Youngblood V, Lee K, Boothby M et al. Regulation of endothelial cell proliferation and vascular assembly through distinct mTORC2 signaling pathways. Mol Cell Biol 2015; 35: 1299-1313.

47. Degtyarev M, De Maziere A, Orr C, Lin J, Lee BB, Tien JY et al. Akt inhibition promotes autophagy and sensitizes PTEN-null tumors to lysosomotropic agents. J Cell Biol 2008; 183: 101-116.

48. Wang RC, Wei Y, An Z, Zou Z, Xiao G, Bhagat G et al. Akt-mediated regulation of autophagy and tumorigenesis through Beclin 1 phosphorylation. Science 2012; 338: 956-959.

49. Manning BD, Tee AR, Logsdon MN, Blenis J, Cantley LC. Identification of the tuberous sclerosis complex-2 tumor suppressor gene product tuberin as a target of the phosphoinositide 3-kinase/akt pathway. Mol Cell 2002; 10: 151-162.

50. Inoki K, Li Y, Zhu T, Wu J, Guan KL. TSC2 is phosphorylated and inhibited by Akt and suppresses mTOR signalling. Nat Cell Biol 2002; 4: 648-657.

51. Rubinsztein DC, Codogno P, Levine B. Autophagy modulation as a potential therapeutic target for diverse diseases. Nat Rev Drug Discov 2012; 11: 709-730.

52. Jia G, Cheng G, Gangahar DM, Agrawal DK. Insulin-like growth factor-1 and TNF-alpha regulate autophagy through $\mathrm{c}$-jun $\mathrm{N}$-terminal kinase and Akt pathways in human atherosclerotic vascular smooth cells. Immunol Cell Biol 2006; 84: 448-454.

53. Keller CW, Fokken C, Turville SG, Lunemann A, Schmidt J, Munz C et al. TNF-alpha induces macroautophagy and regulates MHC class II expression in human skeletal muscle cells. J Biol Chem 2011; 286: 3970-3980.

54. Lin NY, Beyer C, Giessl A, Kireva T, Scholtysek C, Uderhardt S et al. Autophagy regulates TNFalpha-mediated joint destruction in experimental arthritis. Ann Rheum Dis 2013; 72 : 761-768.

55. Tsujimoto Y, Shimizu S. Another way to die: autophagic programmed cell death. Cell Death Differ 2005; 12(Suppl 2): 1528-1534

56. Scott RC, Juhasz G, Neufeld TP. Direct induction of autophagy by Atg1 inhibits cell growth and induces apoptotic cell death. Curr Biol 2007; 17: 1-11. 
57. Alex P, Zachos NC, Nguyen T, Gonzales L, Chen TE, Conklin LS et al. Distinct cytokine patterns identified from multiplex profiles of murine DSS and TNBS-induced colitis. Inflamm Bowel Dis 2009; 15: 341-352.

58. Rogler G, Andus T. Cytokines in inflammatory bowel disease. World J Surg 1998; 22: 382-389.

59. Neal CL, Xu J, Li P, Mori S, Yang J, Neal NN et al. Overexpression of 14-3-3zeta in cancer cells activates PI3K via binding the p85 regulatory subunit. Oncogene 2012; 31: 897-906.

60. Lim GE, Albrecht T, Piske M, Sarai K, Lee JT, Ramshaw HS et al. 14-3-3zeta coordinates adipogenesis of visceral fat. Nat Commun 2015; 6: 7671.

61. Neal CL, Yu D. 14-3-3zeta as a prognostic marker and therapeutic target for cancer. Exp Opin Ther Targets 2010; 14: 1343-1354.

62. Manning BD, Cantley LC. AKT/PKB signaling: navigating downstream. Cell 2007; 129: $1261-1274$.
63. van Gorp AG, Pomeranz KM, Birkenkamp KU, Hui RC, Lam EW, Coffer PJ. Chronic protein kinase $B$ (PKB/c-akt) activation leads to apoptosis induced by oxidative stress-mediated Foxo3a transcriptional up-regulation. Cancer Res 2006; 66: 10760-10769.

64. Stein RC. Prospects for phosphoinositide 3-kinase inhibition as a cancer treatment. Endocr Relat Cancer 2001; 8: 237-248.

65. Workman P. Inhibiting the phosphoinositide 3-kinase pathway for cancer treatment. Biochem Soc Trans 2004; 32(Pt 2): 393-396.

66. Festing MF, Altman DG. Guidelines for the design and statistical analysis of experiments using laboratory animals. ILAR J 2002; 43: 244-258.

67. Nava P, Laukoetter MG, Hopkins AM, Laur O, Gerner-Smidt K, Green KJ et al. Desmoglein2: a novel regulator of apoptosis in the intestinal epithelium. Mol Biol Cell 2007; 18: 4565-4578.

Supplementary Information accompanies this paper on Cell Death and Differentiation website (http://www.nature.com/cdd) 